\title{
A Simple Approach For RELATIVEly AutOMATED HipPOCAMPUS SEGMENTATION FROM SAGITTAL VIEW OF BRAIN MRI
}

\author{
S.Vijayalakshmi, Prabha S. Nair and P.Durgadevi \\ School of Computing Science and Engineering, Galgotias University, Greater Noida, \\ Uttar Pradesh
}

\begin{abstract}
In this paper, we present a relatively automated method to segment the hippocampus in t1 weighted magnetic resonance images that can be acquired in the routine clinical setting. This paper describes a simple approach for segmenting the hippocampus automatically from sagittal view of brain MRI. Large datasets of structural MR images are collected to quantitatively analyze the relationships between brain anatomy, disease progression, treatment regimens, and genetic influences upon brain structure..This method segments the hippocampus without any human intervention for few slices present mid position in the total volume. Experimental results using this method show a good agreement with the manual segmented gold standard. These results may support the clinical studies of memory and neurodegenerative disease.
\end{abstract}

\section{KEYWORDS}

Hippocampus, Alzheimer's disease, neurodegenerative, MRI, segmentation, sagital view.

\section{INTRODUCTION}

Segmentation of medical images is the task of partitioning the data into contiguous regions representing individual anatomical objects. It is a prerequisite for further investigations in many computer-assisted medical applications, e.g. individual therapy planning and evaluation, diagnosis, simulation and image guided surgery. The hippocampus is a complex brain region primarily responsible for memory and any of its associated neurodegenerative diseases such as Alzheimer's disease. Volume and size of the hippocampus are of great importance in the analysis of neurodegenerative diseases. Hippocampus segmentation and its subfield segmentation are playing important roles in the treatment of neurodegenerative diseases[1].

Chupin et al. [2],[3] used a Markovian deformation process with a deformable constraint based on prior knowledge, which is calculated with manual intervention. A user must manually define bounding boxes and create seed points to make algorithm to work and thus making the entire process as semi-automated.

A study by Ashton et al [4] use elastic deformable model with seed points and constraints to segment the hippocampus. The seed points and the constraint were produced from boundaries that had previously been traced manually. These methodologies speed up processing time of segmentation considerably compared to manual segmentation, allowing them to process a large number of scans. The necessity of including human involvement still increases processing time, and it provides a subjective element to segmentation as in manual segmentation. 
Wei Wei Lee et al. [5], proposed a semi-automatic hybrid approach for segmentation of Hippocampus. This method combined low-level image processing techniques such as thresholding, hole filling (based on adjacent voxel connectivity), distance transformation, with high level image processing techniques, and termed it as Geometric Deformable Model (GDM) in a sequential pipeline. The operation of the GDM was based on constraint modeling and cost function minimization. The GDM incorporated 5 constraints which were integrated together to form a local cost function (potential function) associated with each vertex in a 3D model.

An assessment for the performance of standard image registration techniques for MRI-based automated segmentation of the hippocampus was presented by O. T. Carmichael et al. [6]. The study was based on elderly subjects with Alzheimer's Disease as well as mild cognitive impairment (MCI). They have collected structural MR images from Alzheimer's Disease Research Center at the University of Pittsburgh. The subjects were of 54 years and gendermatched healthy individuals, with probable AD, and another set with MCI. The hippocampi from the subject images were segmented by the automated segmentation methods using cohort atlases such as AIR [7], SPM [8], FSL [9], and the fully-deformable method of Chen [10]. The segmented results were aligned to the Harvard atlas [11], MNI atlas [12], and also with manuallylabeled subject images [10].

The segmentation of hippocampus in five patients with mesial temporal sclerosis is described by Robert E. Hogan et al. [13]. Using magnetic resonance (MR) imaging, they verified the precision as well as reproducibility of hippocampal segmentations. They used the deformation based segmentation method. The results produced by them had $92.8 \%$, overall percentage overlap with that of automated segmentations.

In this paper we present an automated tool to segment the hippocampus from high sagittal view of brain MRI . Our method segments the hippocampus in few slices of the entire volume. The remaining part of the paper is organized as follows. In section II, we present the methods and the materials used. In section III, the results and discussion are given. Finally in section IV, the conclusion is given.

\section{Materials AND MethodS}

\section{Materials Used}

The data has been collected from the Whole Brain Atlas [14]. The slices are T1 weighted and in sagittal orientation The hippocampus appears in few slices in the provided atlas. No gold standard is provided for this atlas and it contains only labels for subcortical structures. We have done the manual segmentation with the help of the physicians Dr. C. Kathirvel M.S., M.Ch.(Neuro) and Dr. K. Veni M.D., D.M.(Neuro), Madras Medical College, Chennai..

\section{Methods Used}

The proposed mehtod can segment the hippocampus from the multiple slices for the data set available in WBA [14]. The hippocampus appears only in few slices among 100 slices present in this volume. Figure 1 shows the mid-slice of the brain MRI in the sagittal view obtained from the Whole Brain Atlas [14]. Our method is relatively automated, that it is capable of validating the input slices automatically. The validation process involves checking whether the input slice is suitable for segmentation so that the user can provide any slice with or without HC. Our proposed method validates the input slice by analyzing the image characteristics mean intensity and standard deviation, and then selects the suitable image for segmentation automatically[15-17]. Figure 2 gives the flowchart of our proposed method. 
International Journal on Bioinformatics \& Biosciences (IJBB) Vol.6, No.2, June 2016

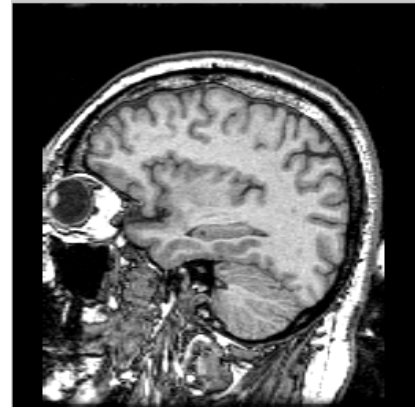

(a)

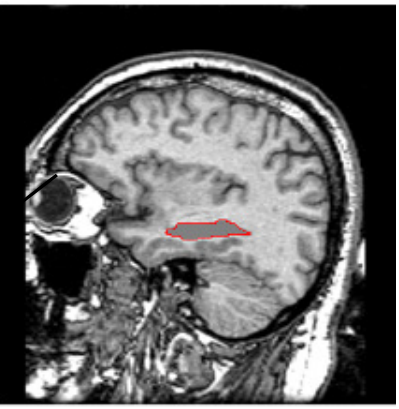

(b)

Figure 1 (a) Mid-slice of brain MRI in sagittal view (b) Hippocampus filled with dark gray color and marked with an arrow

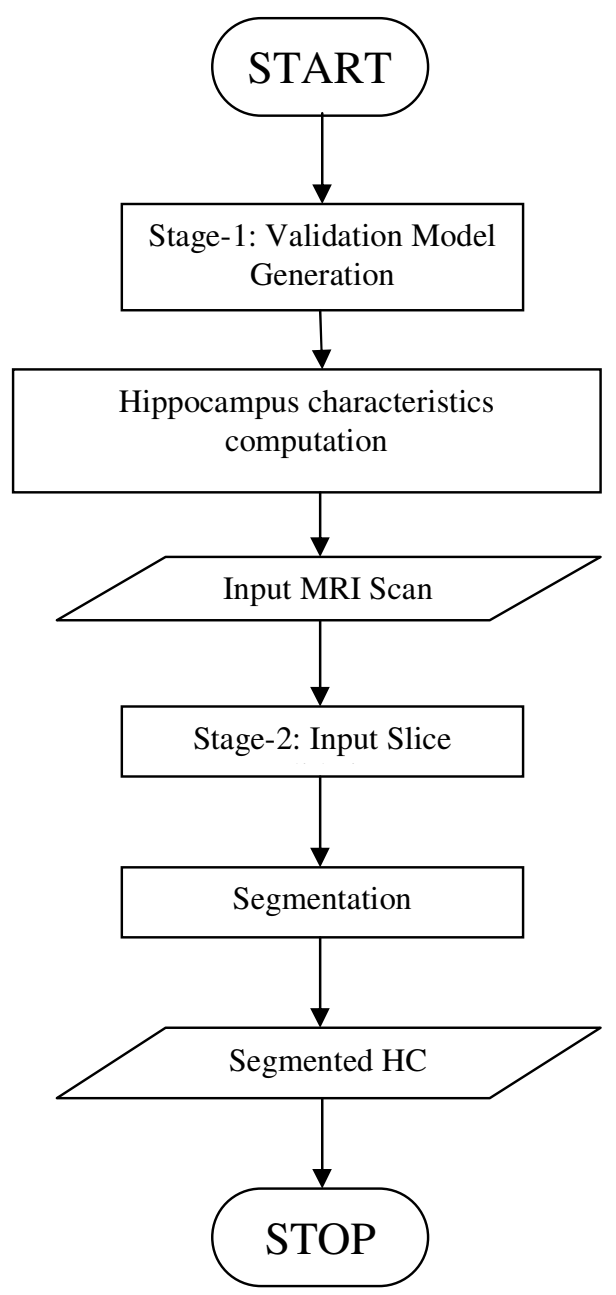

Figure 2 Flowchart of Proposed Method 
International Journal on Bioinformatics \& Biosciences (IJBB) Vol.6, No.2, June 2016

These values are computed from the slices containing $\mathrm{HC}$ available in WBA [89]. The range of values for the valid slices is given in Table 1 .

Table 1. Characteristics of Image with Hippocampus in WBA

\begin{tabular}{|l|l|l|}
\hline Image Characteristic & Characteristic Value \\
\cline { 2 - 3 } & Minimum & Maximum \\
\hline Mean Intensity $(\overline{\boldsymbol{x}})$ & 81.1062 & 88.01 \\
\hline Standard deviation $(\boldsymbol{\sigma})$ & 79.1111 & 88.2 \\
\hline
\end{tabular}

When the input slice is provided by the user, the specified image characteristics $(\bar{X}$ and $\sigma)$ are computed. The mean value $\bar{x}$ of the slice is computed as :

$$
\bar{x}=\frac{\sum_{\mathrm{x}=1}^{\mathrm{m}} \sum_{\mathrm{y}=1}^{\mathrm{n}} \mathrm{f}(\mathrm{x}, \mathrm{y})}{\mathrm{mxn}_{\mathrm{n}}}
$$

where $f(x, y)$ is the intensity of the pixel $A(i, j), m$ is the number of rows and $n$ is the number of columns. The standard deviation of the slice is computed as:

$$
S=\left(\frac{1}{n} \sum_{i=1}^{n}\left(x_{i}-\bar{x}\right)^{2}\right)_{\text {where },}^{\frac{1}{2}} \quad \bar{x}=\frac{1}{n \cdot \sum_{i-1}^{n} x_{i}}
$$

where, $x_{i}$ is the intensity of the pixel at $\mathrm{i}^{\text {th }}$ position.

These values are compared with the values mentioned in Table 1. If the values fall within the specified range, then it is a valid slice and our method proceeds with segmentation process, the input slice is discarded otherwise.

In the next step, the valid slice is enhanced to make the hippocampus prominent and distinguishable with the neighboring region resulting in segmentation of multiple slices. Here two-phase enhancement is implemented in order to make the hippocampus prominent from the neighboring region for multiple slices. The median filter is not suitable for this data set to segment multiple slices. Hence, trimmed mean filter is used for enhancement in addition to the hat operation, which makes the hippocampus boundary distinguished from neighboring pixels, in multiple slices, compared to the first method. The enhanced image is converted to binary image using the block mean threshold as explained in method-1. Binary erosion is used to differentiate the edges of $\mathrm{HC}$ of the binary image. $V \mathrm{H}_{s}$, separated from the neighborhood pixels and is of the form:

$$
H_{s 1}=V H_{s} \Theta \mathrm{D}_{s e}
$$

where, $\mathrm{D}_{s e}$ is a disc shaped structuring element with 17 as radius. The edge detection is done for the eroded image. The Laplacian Gaussian method is used here which has been selected by trial. The other edge detection techniques are unable to detect the edges for the multiple slices in this 
International Journal on Bioinformatics \& Biosciences (IJBB) Vol.6, No.2, June 2016

data set. The middle block contains many connected components other than HC. We use a labeling process as explained in method-1, to assign different labels to each component. The HC is retrieved using the related label which is found by iteration. The HC thus extracted may have lost some of the pixels at the boundary because of the erosion. To recover these pixels, binary dilation is used on $H_{s 1}$, with the same structuring element, which is defined as:

$$
H_{s 2}=H_{s 1} \oplus \mathrm{D}_{s e}
$$

A mask of $\mathrm{HC}$ is obtained after this process. The $\mathrm{HC}$ region is extracted using this mask by mapping it with that of the original input slice $\left(V H_{S}\right)$ as defined by:

$$
H C(i, j)=\left\{\begin{array}{c}
V H_{s}(i, j), \text { if } H_{s 2}(i, j)=1 \\
0, \text { otherwise }
\end{array}\right.
$$

where, $H_{s 2}$ is the mask, containing white pixels as the $\mathrm{HC}$ region and other regions appear as black.

\section{RESULTS AND DISCUSSION}

The computed values of J, D, S, Sp, PA, FPR and FNR are given in Table 2.

Table 2. The computed values of J, D, S, Sp, PA, FPR and FNR for ARAHS

\begin{tabular}{|c|c|c|c|c|c|c|c|}
\hline Slice & $\begin{array}{c}\text { Jaccar } \\
\mathbf{d}\end{array}$ & Dice & $\begin{array}{c}\text { Sensitivit } \\
\mathbf{y}\end{array}$ & $\begin{array}{c}\text { Specificit } \\
\mathbf{y}\end{array}$ & $\begin{array}{c}\text { Predictiv } \\
\mathbf{e} \\
\text { accuracy }\end{array}$ & FPR & FNR \\
\hline $\begin{array}{c}\text { WBA- } \\
\text { sagittal \#95 }\end{array}$ & 0.9997 & 0.9998 & 0.9998 & 0.8772 & 99.968 & 0.0001 & 0.0002 \\
\hline $\begin{array}{c}\text { WBA- } \\
\text { sagittal \#94 }\end{array}$ & 0.9994 & 0.9997 & 0.9995 & 0.9524 & 99.942 & 0.0000 & 0.0005 \\
\hline $\begin{array}{c}\text { WBA- } \\
\text { sagittal \#93 }\end{array}$ & 0.9986 & 0.9993 & 0.9997 & 0.6507 & 99.8596 & 0.0011 & 0.0003 \\
\hline $\begin{array}{c}\text { WBA- } \\
\text { sagittal \#92 }\end{array}$ & 0.9977 & 0.9988 & 1 & 0.5664 & 99.7742 & 0.0023 & 0.0000 \\
\hline $\begin{array}{c}\text { WBA- } \\
\text { sagittal \#91 }\end{array}$ & 0.997 & 0.9985 & 1 & 0.5523 & 99.6994 & 0.0030 & 0.0000 \\
\hline $\begin{array}{c}\text { WBA- } \\
\text { sagittal \#90 }\end{array}$ & 0.9985 & 0.9992 & 0.9998 & 0.7276 & 99.855 & 0.0013 & 0.0002 \\
\hline $\begin{array}{c}\text { WBA- } \\
\text { sagittal \#89 }\end{array}$ & 0.9991 & 0.9995 & 0.9994 & 0.928 & 99.9084 & 0.0003 & 0.0006 \\
\hline AVERAGE & $\mathbf{0 . 9 9 8 6}$ & $\mathbf{0 . 9 9 8 5}$ & $\mathbf{0 . 9 9 9 7}$ & $\mathbf{0 . 7 5 0 7}$ & $\mathbf{9 9 . 8 5 8 1}$ & $\mathbf{0 . 0 0 1 2}$ & $\mathbf{0 . 0 0 0 3}$ \\
\hline
\end{tabular}




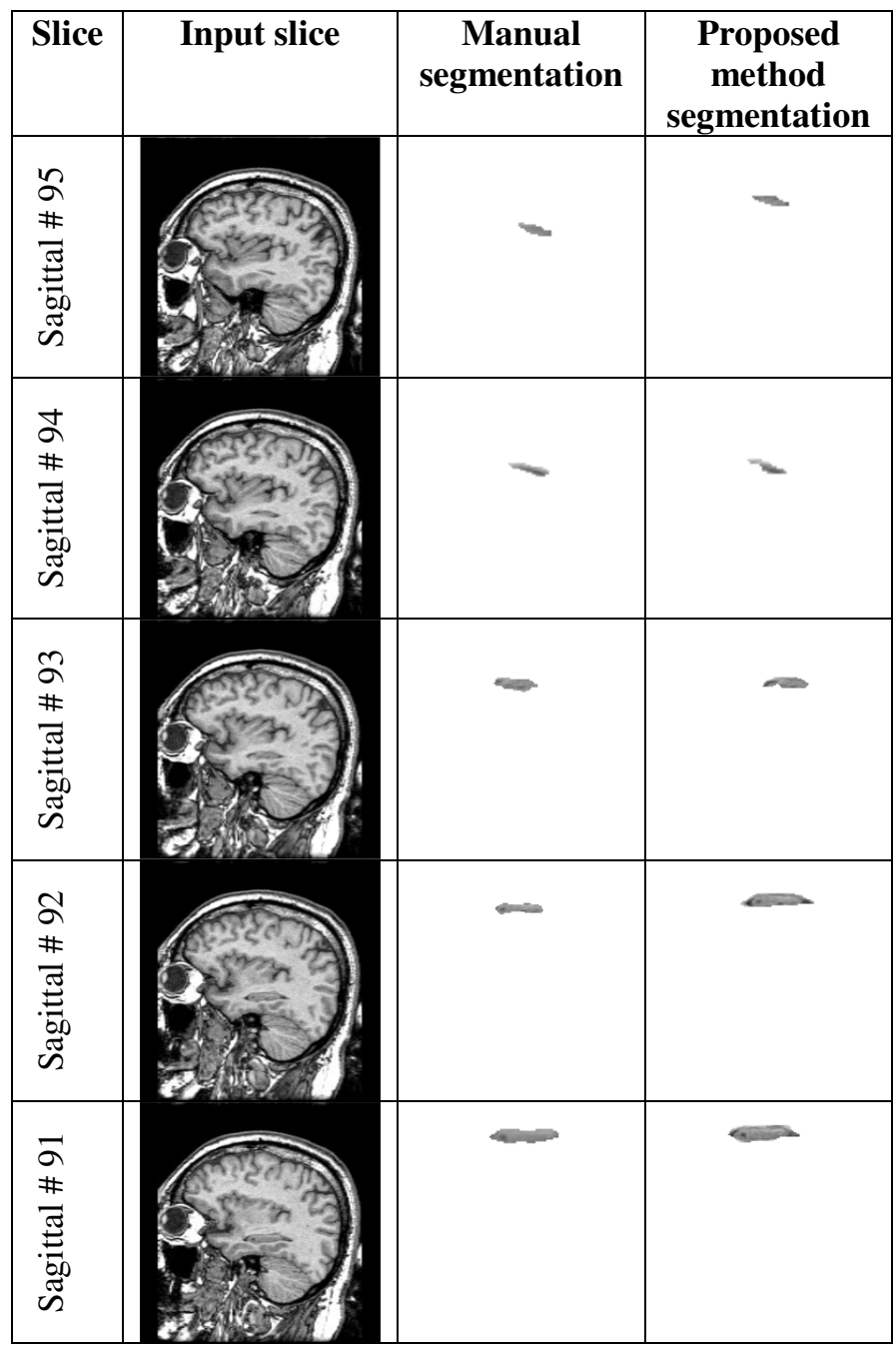

Figure 2. Segmented results of ARAHS: first column shows the input slice, second column shows the manually segmented hippocampus and third column contains the segmented hippocampus by proposed method

From Table 2, we observe that the average of Jaccard index values is $\mathbf{0 . 9 9 8 6}$ and the Dice coefficient is $\mathbf{0 . 9 9 8 5}$. This implies that our segmented results are better. The sensitivity value is closer to 1 and predictive-accuracy is very close to 100. The FPR and FNR values are nearly equal to zero. Hence our proposed method segments the hippocampus nearly equal to the gold standard. The segmented results are shown in Figure 2.

\section{CONCLUSiON}

Our proposed method uses only simple techniques to extract the hippocampus from the sagittal view of the brain MRI. This method is developed in a motive of employing simple image processing techniques to segment the hippocampus from MRI head scans in different orientations. The strengths of these methods are their simplicity, automation and reduction of computation time. 


\section{REFERENCES}

[1] Alzheimer's Association, 2012 Alzheimer's Disease Facts and Figures, Alzheimer's \& Dementia, Volume 8, Issue 2.

[2] Chupin, M.,A. Hammers, A. Bardinet E, Colliot O, Liu R.S.N. and Duncan J.S., 2007, "Fully Automatic Segmentation Of The Hippocampus And The Amygdala From MRI Using Hybrid Prior Knowledge", Med Image Comput Assist Interv, Vol.10, pp.875-82.

[3] Chupin M, Mukuna-Bantumbakulu A. R., Hasboun D., Bardinet E., Baillet S., Kinkingnehun S., Lemieux L., Dubois B. and Garnero L., 2007, “Anatomically Constrained Region Deformation For The Automated Segmentation Of The Hippocampus And The Amygdala: Method And Validation On Controls And Patients With Alzheimer's Disease", Neuroimage, Vol.34(3), pp.996-1019.

[4] Ashton E., K. Parker, Berg M. and Chen C, 1997, "A novel volumetric feature extraction technique with applications to MR images", IEEE Trans Med Imaging, Vol.6(4), pp. 365-71.

[5] Wei Wei Lee, Iain Richardson, Ken Gow, Yafan Zhao and Roger Staff, 2005, "Hybrid Segmentation of the Hippocampus in MR Images", Proceedings of European Signal Processing Conference $-13^{\text {th }}$ Series.

[6] Owen T. Carmichael, Howard A. Aizebstein, Simon W. Davis, James T. Becker, Paul M. Thompson, Carolyn Cidis Meltzer and Yanxi Liu, 2004, "Atlas-Based Hippocampus Segmentation In Alzheimer's Disease and Mild Cognitive Imapirment”, report CMU-RI-TR-04-53, Robotics Institute, Carnegie Mellon University.

[7] Woods R.P., Grafton S.T., Holmes C.J., Cherry S.R., and Mazziotta J.C., 1998 , "Automated image registration: I. general methods and intrasubject, intramodality validation", Journal of Computer Assisted Tomography, Vol.22, pp.139-152.

[8] Friston K.J., Ashburner J., Frith C.D., Poline J.B., Heather J.D. and Frackowiak R.S.J.. 1995, "Spatial registration and normalization of images" In Annual Meeting of the Organization for Human Brain Mapping, pp.165-189.

[9] Mark Jenkinson, Peter Bannister, Michael Brady and Stephen Smith, 2002, "Improved methods for the registration and motion correction of brain images", Technical report, Oxford Centre for Functional Magnetic Resonance Imaging of the Brain.

[10] Mei Chen, 1999, “ 3-D Deformable Registration Using a Statistical Atlas with applications in Medicine”, PhD thesis, Robotics Institute, Carnegie Mellon University, Pittsburgh.

[11] Ron Kikinis, Chiara M. Portas, Robert M. Donnino, Ferenc A. Jolesz, Martha E. Shenton, Dan V. Iosifescu, Robert W. McCarley, Pairash Saiviroonporn, Hiroto H. Hokama, Andre Robatino, David Metcalf and Cynthia G. Wible, 1996, “A digital brain atlas for surgical planning, model-driven segmentation, and teaching”, IEEE Transactions on Visualization and Computer Graphics, Vol.2(3), pp.232-24.

[12] Tzourio-Mazoyer N, Landeau B, Papathanassiou D, Crivello F, Etard O and Delcroix N., 2002, "Automated anatomical labelling of activations in spm using a macroscopic anatomical parcellation of the MNI MRI single subject brain". NeuroImage, Vol.15, pp.273-289.

[13] Robert E. Hogan, Kevin E. Mark, Lei Wang, Sarang Joshi, Michael I. Miller, Richard D. Bucholz, 2000, "Mesial Temporal Sclerosis and Temporal Lobe Epilepsy: MR Imaging Deformation-Based Segmentation of the Hippocampus In Five Patients", Radiology, Vol.216, pp.291-297.

[14] Johnson K.A. and Becker A.J., 2012, The Whole Brain Atlas - a research and educational site of actual human brains, Last accessed http://www.med.harvard.edu/AANLIB/home.html.

[15] Dr.K.Somasundaram and Ms. S.Vijayalakshmi, (2012), "Hippocampus Segmentation Based On Priori Shape Model with Region Growing From High-Resolution MRI Of Post-Mortem Samples", International journal of Advanced Research in Computer Science, Vol.3, pp.320-324.

[16] Dr.K.Somasundaram and Ms. S.Vijayalakshmi , (2012), “A Simple Automatic Tool For Segmenting the Right Hippocampus from Mid-Slices of Brain MRI”, International Journal of Scientific Research and Publications, Vol.2, pp.1-7.

[17] Dr.K.Somasundaram and Ms. S.Vijayalakshmi, (2012), "A Semi-Automatic Atlas-Based Method for Segmentation of the Hippocampus from the mid slices of brain MRI", International Journal of Computer Application, Vol.5, pp.32-41. 


\section{Authors}

Vijayalakshmi S was born in the year 1975. She received the B.Sc. degree in Computer Science from Bharathidasan University, Trichirapalli, India in 1995, the MCA degree from the same University in 1998 and the M.phil. degree from the same University in the year 2006. She received her doctorate in 2013. She has been working as an Assistant Professor (grade-III), Galgotias University, Greater Noida, Uttar Pradesh, India she has 17 years of teaching experience and 10 years of research experience. She has published many papers in the area of image processing especially in medical imaging

Prabha Shreeraj Nair was born in chhattisgarh, India, in 1973. She received the B.E. Degree in Computer Technology from Nagpur University, in 1996 and Masters in Computer Science and Engineering from Kakatiya University, in 2007. She has been working as an Assistant Professor, Galgotias University, Greater Noida, Uttar Pradesh, India she has 18 years of teaching experience. She is engaged in research on Software Engineering, Augmented reality.

Durgadevi P was born in Kallakurichi, India, in 1986. She received the B.E. Degree in Electronics and Communication Engineering from Anna University Chennai, in 2008 and Masters in Computer Science and Engineering from Anna University, in 2010. In 2010, she joined as an Assistant Professor in Department of Information Technology, Vivekanandha Institute of Engineering College for women. Since May 2014, she has been with the School of Computing Science and Engineering as an Assistant Professor, Galgotias University, Uttar Pradesh. She is engaged in research on Bio-

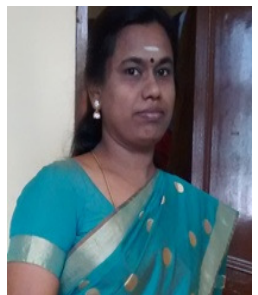
medical electronics and image Processing. 